\title{
Reducción en la fecundidad en Manfreda brachystachya (Cav.) Rose, una agavácea polinizada por murciélagos: los riesgos de la especialización en la polinización
}

El presente trabajo pretende explicar la reducción de $75 \%$ en la fecundidad, en Manfreda brachystachya entre los años de 1982 y 1985, en términos de un descenso en las poblaciones de su polinizador más importante, el murciélago nectarívoro Leptonycteris sanborni.

Manfreda brachystachya crece en lugares moderadamente secos y perturbados del centro de México. Aspectos de su ecología y biología reproductiva se encuentran en Eguiarte (1983) y Eguiarte y Búrquez (1987). Hemos estudiado plantas en los alrededores del Jardín Botánico Exterior de la UNAM, de la ciudad de México en un cuadro de $10 \times 10$ $\mathrm{m}$ en 1982 y en uno de $50 \times 50$ m, que incluía al de 1982, en 1985. En tres años el cociente del número de frutos producido entre el número de flores producidas (cociente de producción de frutos) se redujo significativamente de 0.1913 ( \pm 0.125 desviación estándar) $(n=60)$, en 1982 a $0.485 \pm 0.0865(n=93)$ en 1985. Las dos producciones de frutos son significativamente distintas (prueba de t entre datos transformados arcoseno $\mathrm{t}=3.787, \mathrm{P}<0.001)$.

M. brachystachya está fundamentalmente limitada en su producción de frutos por polinizadores, dado que polinizaciones artificiales siempre aumentan la producción de frutos (en $25 \%$ en promedio) y que prácticamente no se abortan frutos iniciados (la pérdida de frutos por aborción y por depredación predispersión sumadas fue de menos de 2\% en 1982) (Eguiarte y Búrquez, 1987).

Dado que la fecundidad está limitada por polinizadores, una reducción mayor significa que disminuyen el número o la actividad de los polinizadores. $M$. brachystachya es polinizada por gran cantidad de animales, tanto diurnos como nocturnos (Eguiarte y Búrquez, 1987). Para evaluar la importancia relativa de los distintos polinizadores, en 1983 se realizaron los siguientes experimentos: se cubrieron flores de día y se dejaron descubiertas la noche de mayor receptividad $(n=33)$ y se cubrieron otras flores de noche y se dejaron descubiertas el día de mayor receptividad $(n=28)$. Otras flores se dejaron descubiertas tanto de día como de noche para funcionar como controles $(n=10)$. Los resultados indican que los polinizadores más eficientes eran los nocturnos, con un cociente de producción de frutos de 0.273 , mientras que los diurnos produjeron 0.071 y los controles 0.30 . Por observaciones directas se encontró que el polinizador nocturno más importante era el murciélago nectarívoro Leptonycteris sanborni, y mucho menos abundantes, los murciélagos nectarívoros L. nivalis y Anoura geoffroyi. Algunas polillas (principalmente esfíngidos) tal vez polinicen, pero sus visitas son muy raras. De día las flores son fundamentalmente polinizadas por cuatro especies de colibríes y su menor eficiencia se debe a que al visitar las flores no siempre contactan las partes reproductivas y a que abejas (fundamentalmente Apis mellifera), colectan eficiente y rápidamente el polen por las mañanas.

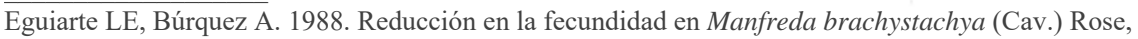
una agavácea polinizada por murciélagos: los riesgos de la especialización en la polinización. Boletín de la Sociedad Botánica de México 48: 147-149. 
Por lo anterior, la reducción en la producción de frutos en $M$. brachystachya se debe a una reducción en la actividad o en el número de los colibríes o de los murciélagos polinizadores. Las poblaciones de los colibríes polinizadores parecen abundantes, y cuando menos dos, debido a sus hábitos "ruderales", son muy abundantes en la ciudad de México (Hylocharis leucotis y Cynanthus latirostris) (L. Eguiarte, observación personal).

Para los murciélagos, y concretamente para Leptonycteris spp. la situación es muy distinta. En el suroeste de Estados Unidos se encontró que las poblaciones de L. sanborni han pasado de decenas de miles a unos 135 animales de 1970 a 1980 (Howell y Roth, 1981). Esta reducción en los murciélagos ocasionó una fuerte reducción en el cociente de producción de frutos de Agave parryi de 0.9 en 1935 a 0.04 en 1976. En una cueva de Texas (Emory Peak), L. nivalis descendió de 10650 individuos en 1967 a 1000 en 1983 (Wilson et al., 1985). Colectas intensivas en 1983, en Coahuila, Nuevo León, San Luis Potosí y Tamaulipas sólo ofrecieron resultados de 20 Leptonycteris (15 L. nivalis y 5 L. sanborni) y observaciones de menos de 100 individuos, aunque en una sola cueva de la zona se colectaron 100 L. nivalis en 1941 (Wilson et al. 1985). También en el centro de México se ha observado una paulatina disminución en las poblaciones de L. sanborni y nivalis (B. Villa, H. Arita y R. Medellín, comunicación personal). En las cuevas del centro de México habitadas por Leptonycteris spp., que cita Villa (1967), actualmente ya no existen poblaciones de estos murciélagos. No se cuenta con evidencia directa de reducciones en el número de Leptonycteris spp., pero dado el carácter migratorio de las dos especies (Wilson et al., 1985) se debe esperar que una reducción demostrada en parte de su distribución se refleje como una reducción en otros lugares. Por otra parte, en 1981 y en 1982 se observaron murciélagos nectarívoros en inflorescencias de Agave spp. en el Jardín Botánico Exterior, UnAm (A. Búrquez y C. Martínez del Río, datos no publicados). Las visitas eran de más de 100 por hora, y la especie más común era L. sanbor ni. En 1985 se repitieron estas observaciones (C. Martínez del Río y L. Eguiarte, datos no publicados) pero el número de visitas fue de menos de 20 por hora.

Aparentemente la reducción en la fecundidad de M. brachystachya en 1985 se debe a una reducción en el número de murciélagos polinizadores. Por otra parte, el cociente de producción de frutos (los frutos producidos entre flores producidas) en 1985 no es significativamente distinto al que produjeron exclusivamente los polinizadores diurnos en 1982 (prueba de $t$ entre datos transformados arcoseno $t=0.154, \mathrm{P}>0.8$ ).

La presente nota pretende llamar la atención para que se realicen, por un lado, estudios sobre las poblaciones de murciélagos nectarívoros, y por otro estudios sobre la producción de semillas en las plantas por ellos polinizadas.

Por último, como la reducción en la fecundidad fue de $75 \%$, un mutante que se autopolinizara o fuera eficientemente polinizado por colibríes o esfíngidos dejaría más semillas. Si hubiera variabilidad genética en este carácter, se esperaría que por selección natural la población cambiara su biología floral. Podríamos considerar esta reducción en el número de murciélagos como un experimento que nos puede aportar datos sobre cómo opera el proceso de la adaptación.

Agradecimientos. Agradecemos a Betty Benrey, que nos ayudó en el campo en 1985. A Héctor Arita agradecemos sus amplias pláticas y discusiones sobre Leptonycteris. Daniel Piñero, Ken Oyama, Valeria Souza, Héctor Arita, Carlos Cordero, Rodolfo Dirzo, César Domínguez 
y Juan Núñez leyeron una primera versión del presente manuscrito. Carlos Martínez del Río colaboró con discusiones y observaciones en distintas fases de este trabajo.

\section{LITERATURA CITADA}

Eguiarte, L.E. 1983. Biología floral de Manfreda brachystachya (Cav.) Rose en el Pedregal de San Ángel, D.F. Tesis profesional, Facultad de Ciencias, UNAM.

, y A. BúRQUEZ, 1987. Reproductive ecology of Manfreda brachystachya, an iteroparous agavaceae. Southwest. Nat. 32:169-178.

Howell, D.J. y B.S., ROTH, 1981. Sexual reproduction in agaves: the benefits of bats; the cost of semelparous advertising. Ecology 62:1-7.

Wilson, D.E., R.A., Medelí́n, D.V. Lanning y H.T. Arita, 1985. Los murciélagos del noroeste de México, con una lista de especies. Acta Zool. 8:1-26.

Villa-R., B., 1967. Los murciélagos de México. Instituto de Biología, UNAM. México.

Luis E. Egutarte y Alberto Búrquez. Depto. de Ecología, Instituto de Biología, unam. Apartado Postal 70-233, México D.F. Coyoacán 04510. México. Dirección actual: Centro de Ecología, Universidad Nacional Autónoma de México, Apdo. Postal 70-275, 04510 México, D.F. 